\title{
PRODUZIONE ACCADEMICA ED EDUCATIVA DI UNO STUDENTE MAGISTRALE IN EPT OF IFAP MACAPÁ, AMAPÁ, AMAZÔNIA, BRASILE
}

\section{ARTICOLO ORIGINALE}

VIANA, Valderi Nascimento ${ }^{1}$, FECURY, Amanda Alves², OLIVEIRA, Euzébio de ${ }^{3}$, DENDASCK, Carla Viana ${ }^{4}$, DIAS, Claudio Alberto Gellis de Mattos ${ }^{5}$

VIANA, Valderi Nascimento. Et al. Produzione accademica ed educativa di uno studente magistrale in EPT Of IFAP Macapá, Amapá, Amazônia, Brasile. Revista Científica Multidisciplinar Núcleo do Conhecimento. Anno 06, Ed. 06, Vol. 08, pp. 186200. Giugno 2021. ISSN: 2448-0959, Link di accesso: https://www.nucleodoconhecimento.com.br/formazione-it/studente-magistrale, DOI: 10.32749/nucleodoconhecimento.com.br/formazione-it/studente-magistrale

\section{RIEPILOGO}

La produzione scientifica all'interno di corsi di laurea e laurea ha la sua importanza per la costruzione di un nuovo modo di pensare dello studente. II Master Professionale in Formazione Professionale in Rete Nazionale (ProfEPT) unisce contenuti disciplinari, conoscenze pedagogiche e produzione accademica finale e li collega a situazioni reali di pratica educativa, e il lavoro finale può essere presentato in vari modi (articoli, libri, prodotti educativi). La produzione accademica, sia del consulente che della guida, oltre

\footnotetext{
${ }^{1}$ Educatore fisico, specialista in fisiologia dell'esercizio fisico e nutrizione sportiva (FAMA), studente del corso di laurea in educazione professionale e tecnologica (PROFEPT IFAP).

${ }^{2}$ Biomedicale, PhD in Malattie Tropicali, Professore e ricercatore del Corso di Medicina del Campus Macapá, Università Federale di Amapá (UNIFAP).

${ }^{3}$ Biologo, PhD in Malattie Tropicali, Professore e ricercatore del Corso di Educazione Fisica dell'Università Federale di Pará (UFPA).

${ }^{4}$ Teologo, Dottorato di Ricerca in Psicoanalisi Clinica. Lavora da 15 anni con metodologia scientifica (metodo di ricerca) nella guida alla produzione scientifica di studenti di master e dottorato. Specialista in Ricerche di Mercato e Ricerca focalizzato sulla salute. Dottorando in Comunicazione e Semiotica (PUC SP).

${ }^{5}$ Biologo, Dottore di Ricerca in Teoria e Comportamento, Professore e ricercatore del Corso di Laurea in Chimica dell'Istituto di Educazione Di Base, Tecnica e Tecnologica di Amapá (IFAP) e del Corso di Laurea in Formazione Professionale e Tecnologica (PROFEPT IFAP).
}

RC: 89149

Link di accesso: https://www.nucleodoconhecimento.com.br/formazione-it/studentemagistrale 
allimportanza legata alla divulgazione scientifica, ha peso nella valutazione del programma e anche nella sua manutenzione. L'obiettivo di questo lavoro è quello di mostrare quantitativamente la produzione accademica ed educativa di uno studente di master in Educazione Professionale e Tecnologica (EPT) dell'Istituto di Istruzione, Scienza e Tecnologia (IFAP) di Macapá, Amapá, Amazônia, Brasile. Durante il master, la produzione scientifica in varie forme si è rivelata necessaria durante il processo di formazione. Unire la teoria alla pratica della scrittura, ha facilitato la produzione della tesi che dovrebbe essere presentata alla fine del corso. Attraverso il processo di organizzazione, la pianificazione delle idee ha portato a un nuovo pensiero strutturato secondo gli standard dell'accademia, ma che non si limita a questo spazio e alle sue norme. La produzione durante il master cerca di contribuire sia quantitativamente che qualitativamente alla produzione scientifica della regione settentrionale, imprevolandosi positivamente e con il programma del master. Oltre all'importanza nel contributo della creazione e diffusione scientifica, le produzioni tendono ad avere un impatto positivo sulla valutazione e sul mantenimento del programma PROFEPTIFAP, svolto da CAPES.

Parole chiave: EPT, Divulgazione, Produzione Scientifica, PRORFEPT.

\section{INTRODUZIONE}

Unendo strumenti teorici e metodologici opportunità di realizzazione di nuovi progetti, lo studente cessa di essere solo un ripetitore e inizia a produrre conoscenza, contribuendo con un tema o un'area specifica. Attraverso la costruzione del proprio pensiero, producono nuovi atteggiamenti e comportamenti, cioè espone un'idea plausibile e strutturata, presentando giudizi di valore e senso critico con un'argomentazione valida e logica (Maia, 2008).

II Master Professionale in Formazione Professionale in Rete Nazionale (ProfEPT) emerge come un'opportunità di formazione specifica sull'Educazione Professionale e Tecnologica sia per i dipendenti degli Istituti Federali che per la popolazione esterna, avendo la produzione di conoscenze attraverso prodotti educativi e risultati di ricerca 
che riguardano il mondo del lavoro. In questa modalità, l'obiettivo è l'unione di contenuti disciplinari e conoscenze pedagogiche, la produzione accademica finale deve essere collegata a situazioni reali di pratica educativa, e il lavoro finale può essere presentato in vari modi (Machado e Urbanetz, 2019; Urbanetz et al., 2020).

Per la produzione e la pubblicazione di conoscenze scientifiche, l'orientamento svolge un ruolo fondamentale. II consulente con una vasta esperienza nell'editoria, diventa un mentore per il suo mentoring, influenzando positivamente la produttività, essendo responsabile dell'apprendimento del progresso e del successo nel futuro dello studente. Cioè, tutta l'esperienza acquisita durante il processo addestramento ha un impatto positivo sulla vita accademica dello studente, le competenze e le competenze per la pubblicazione vengono apprese durante la formazione (Falaster et al., 2017).

Secondo il modulo di valutazione per i programmi accademici e professionali (Area 46: Insegnamento) del Coordinamento per il Miglioramento del Personale dell'Istruzione Superiore (CAPES):

[...] sarà valutata la relativa capacità produttiva minima del DP, che può coinvolgere studenti e laureati. Inoltre, verrà valutata l'aderenza e l'allineamento della produzione indicata dal PPG DP (quattro produzioni) con la sua area di concentrazione e le linee di ricerca, riflettendo la coerenza della produzione con gli obiettivi e l'identità del Programma. [...] Per questo, il PPG indicherà fino a cinque produzioni bibliografiche e fino a cinque PTT pubblicate nel quadriennio coinvolgendo studenti e laureati. [...] la pertinenza della produzione indicata in relazione all'impatto e al carattere innovativo della produzione intellettuale, copertura locale, regionale, nazionale o internazionale, e soddisfare le esigenze sociali. (Capes, 2020).

La produzione accademica, sia del consulente che del mentore, oltre all'importanza legata alla divulgazione scientifica, ha peso nella valutazione del programma di laurea stricto sensu e anche nella sua manutenzione. 


\section{OBIETTIVO}

Dimostrare quantitativamente la produzione accademica ed educativa di un master in Educazione Professionale e Tecnologica (EPT) dell'Istituto di Istruzione, Scienza e Tecnologia (IFAP) di Macapá, Amapá, Amazônia, Brasile.

\section{METODOLOGIA}

Questo articolo espone la produzione intellettuale sviluppata durante ProfEPT - IFAP. Queste produzioni che possono essere classificate come produzione bibliografica (articolo in periodico, libro) e produzione tecnica (applicazione - APP, sito web)

Le indagini quantitative per ottenere i dati sull'applicazione e sul sito web hanno utilizzato il periodo dal 12 aprile 2021 al 14 giugno 2021. I dati sull'app sono stati presi dalla Play Console (Playconsole, 2021) e dal sito Web di Google Analytics (Googleanalytics, 2021).

Per i libri e gli articoli scientifici, la data di pubblicazione originale è stata utilizzata fino al 14 giugno 2021. I dati su di essi sono stati presi dai dati online (libri e articoli pubblicati su Revista Multidisciplinar Núcleo do Conhecimento) (Rmnc, 2021) e anche dalla consultazione diretta della rivista (articolo pubblicato su Research, Society and Development journal) (Rsd, 2021).

\section{RISULTATI}

\section{APPLICAZIONE EFE (EDUFISEPT)}

La figura 1 mostra la percentuale di installazioni di applicazioni EFE per fonte di traffico di informazioni tra il 12 aprile e il 14 giugno 2021. I dati mostrano che il 21,67\% (13 utenti) è arrivato all'app attraverso la ricerca all'interno del Play Store di Google e il $78,30 \%$ (47 utenti) tramite link di terze parti. 
Figura 1 - Mostra la percentuale di installazioni di applicazioni EFE per fonte di traffico di informazioni tra il 12 aprile e il 14 giugno 2021.

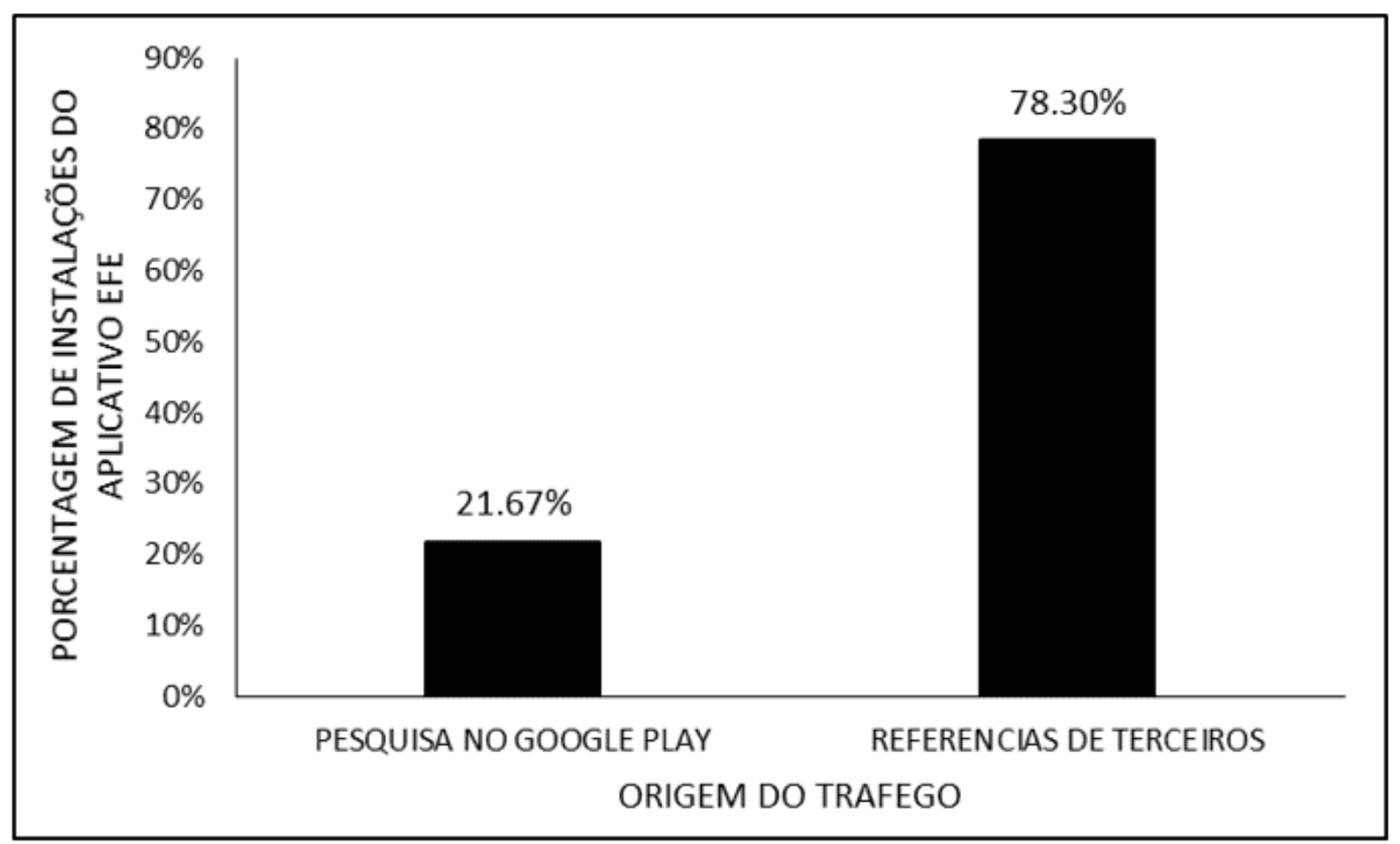

Fonte: Adattato da Playconsole (2021)

Per quanto riguarda l'analisi della percentuale di installazioni dell'applicazione EFE per paese dell'utente, tra il 12 aprile e il 14 giugno 2021 si sono registrati download in due paesi, con il 98,33\% (59 download) dal Brasile e l'1,67\% dal Portogallo (01 download) (Figura 2). 
Figura 2 - Mostra la percentuale di installazioni di app EFE per paese utente tra il 12 aprile e il 14 giugno 2021.

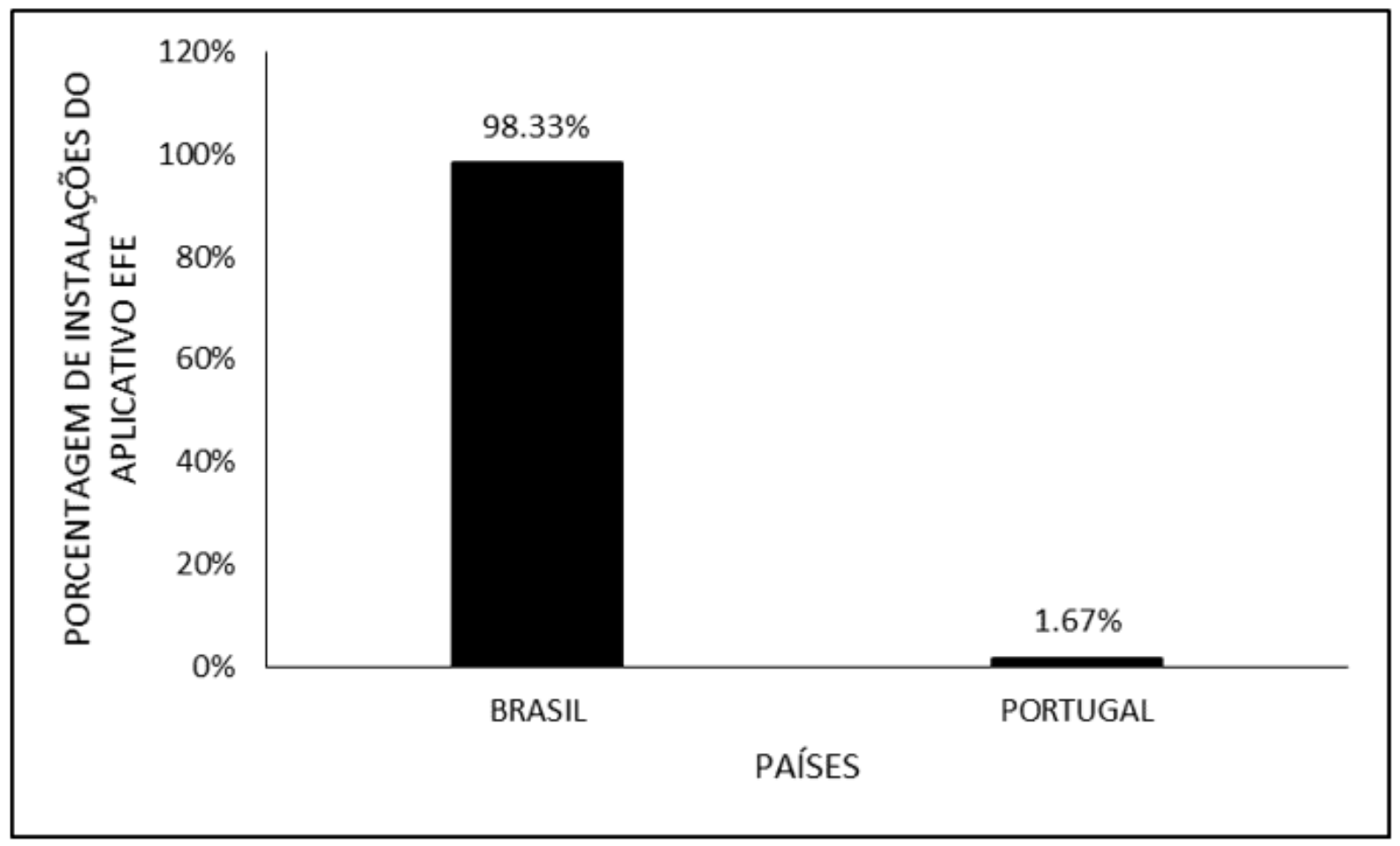

Fonte: Adattato da Playconsole (2021)

La percentuale di installazioni di app EFE per visita e installazione dell'app tra il 12 aprile e il 14 giugno 2021 è illustrata nella Figura 3. Del 100\% dei visitatori della pagina in negozio (82 persone), il 73,17\% (60) persone ha scaricato l'applicazione al proprio dispositivo mobile (Figura 3 ). 
Figura 3 - Mostra la percentuale di installazioni di app EFE per visita e installazione dell'app, tra il 12 aprile e il 14 giugno 2021.

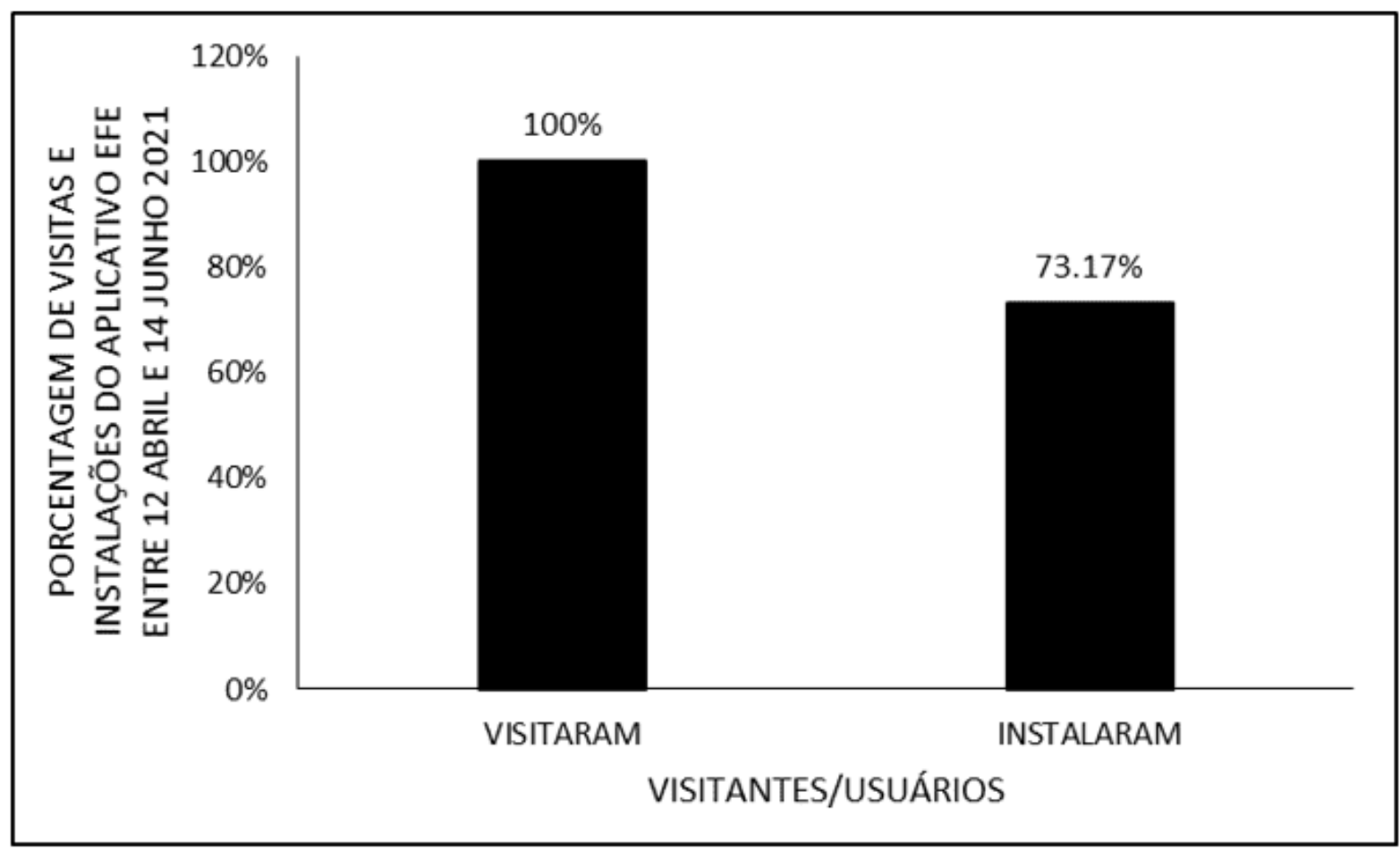

Fonte: Adattato da Playconsole (2021)

La figura 4 mostra la percentuale di installazioni di app EFE per tipi di utente tra il 12 aprile e il 14 giugno 2021. Del totale (60 utenti), il 93,33\% (56 persone) sono nuovi utenti e il 6,67\% (04 persone) sono utenti ricorrenti all'interno dell'applicazione, cioè hanno avuto accesso all'APP più volte. 
Figura 4 - Mostra la percentuale di installazioni di app EFE per tipi di utente tra il 12 aprile e il 14 giugno 2021.

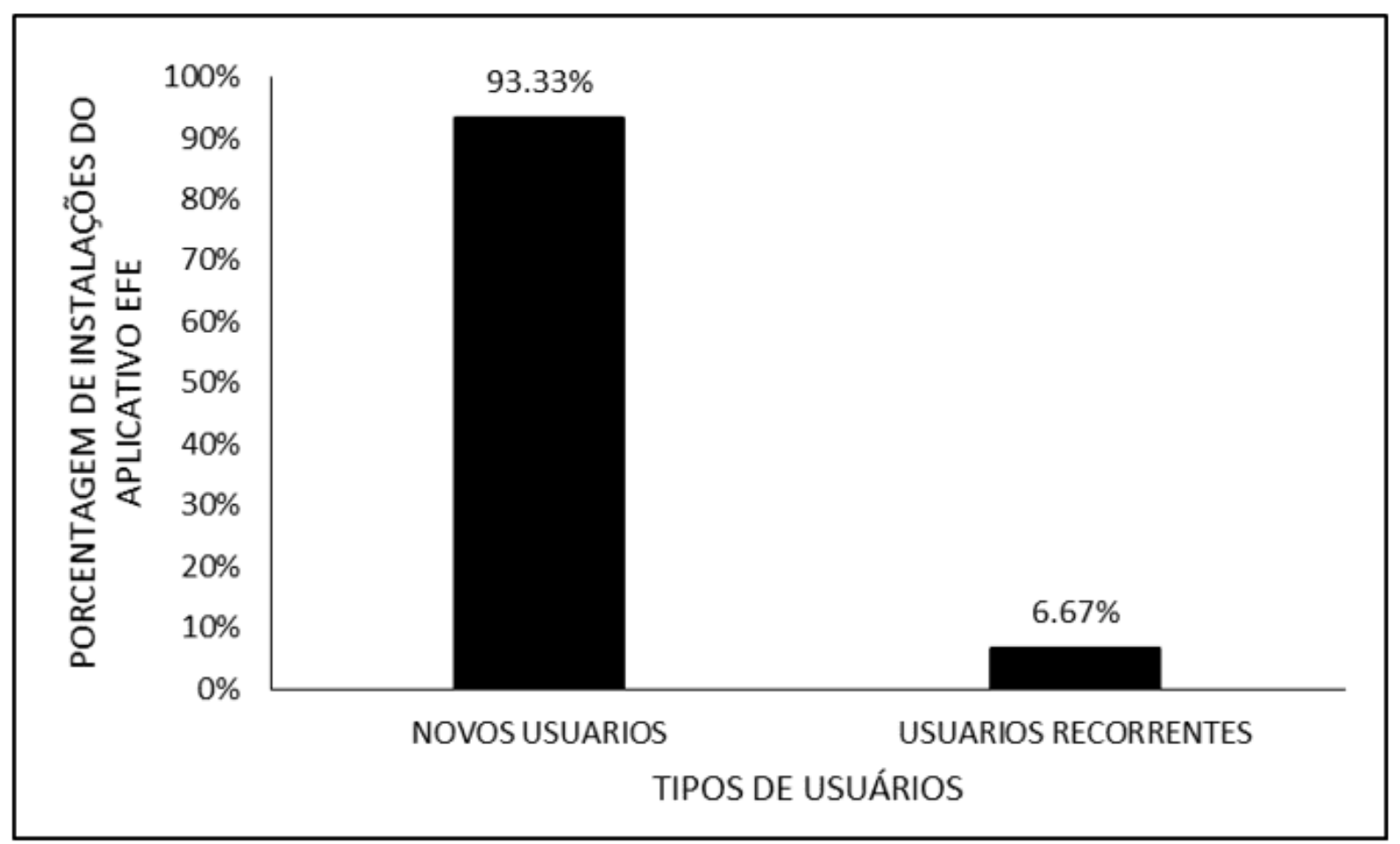

Fonte: Adattato da Playconsole (2021)

\section{SITO}

Per quanto riguarda la percentuale di accessi al sito EduFisEPT da parte delle pagine interne del sito, tra il 12 aprile e il 14 giugno 2021, la pagina con il maggior accesso tra i 1399 utenti (100\%) è pratiche pedagogiche con il 30,38\% (425 persone). Successivamente la Pagina dello Sport con il 17,23\% (241 persone che accedono), Autori con il 13,37\% (187 persone), Collabora con il 13,22\% (185 persone), Giochi e giochi con il 7,79\% (109 persone che accedono), Balli 5,65\% (79 persone), Salute con il 5,00\% (70 persone), Tempo libero con il 4,43\% (62 persone) e Lotte con il 2,91\% (41 persone) (Figura 5). 
Figura 5 - Mostra la percentuale di accessi al sito EduFisEPT per pagina interna tra il 12 aprile e il 14 giugno 2021.

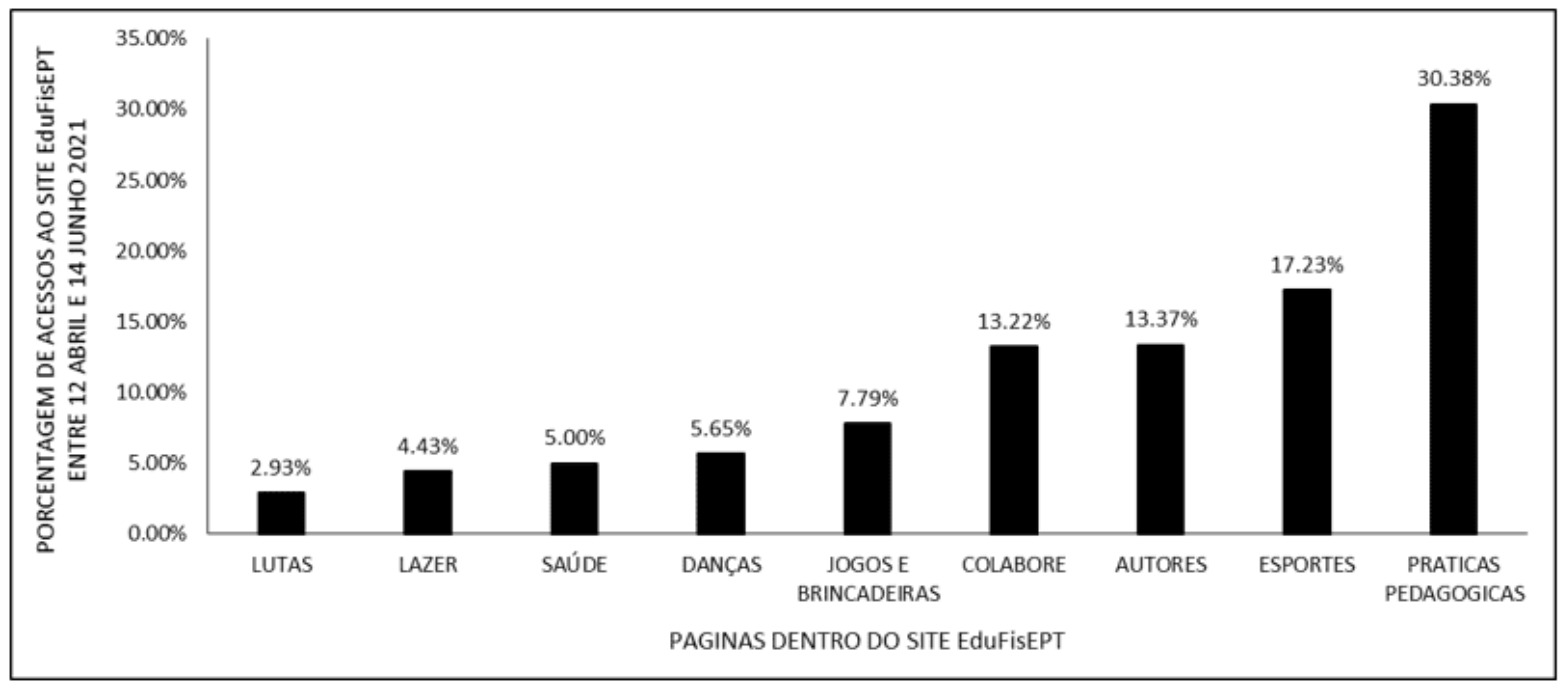

Fonte: Adattato da GoogleAnalytics (2021).

La percentuale di accessi al sito eduFisEPT per paesi, tra il 12 aprile e il 14 giugno, 2021, porta il Brasile con il 93,92\% (1314 persone) accessi, seguito dal Portogallo con il 2,64\% (37 persone), dagli Stati Uniti con I'1,50\% (21 persone), dal Mozambico dall' $1,07 \%$ (15 persone), dall' $1,07 \%$ e dall'Irlanda con lo $0,86 \%$ (12 persone) (figura 6 ). 
Figura 6 - Mostra la percentuale di accessi al sito Web EduFisEPT per paesi tra il 12 aprile e il 14 giugno 2021.

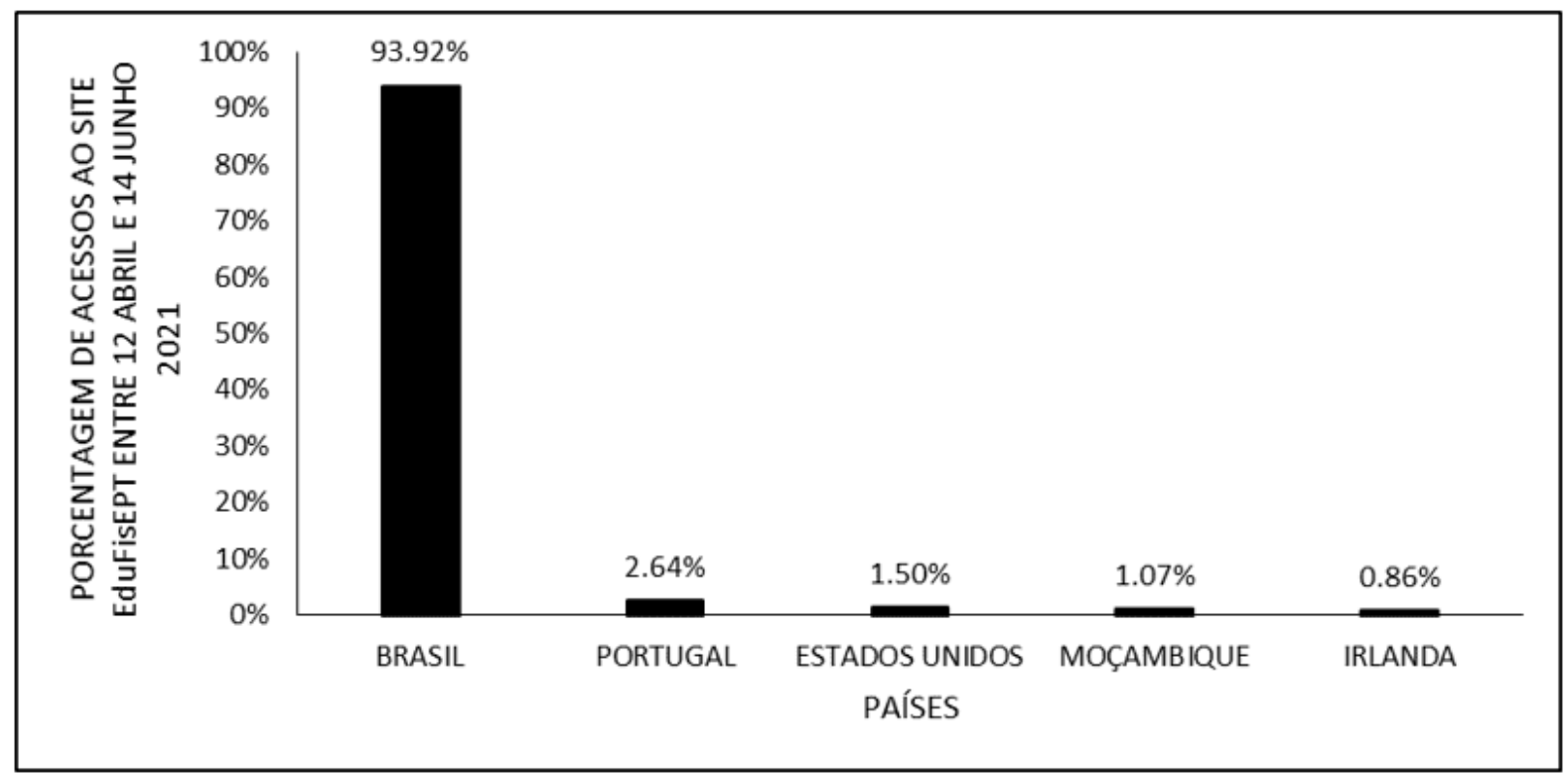

Fonte: Adattato da GoogleAnalytics (2021).

\section{LIBRI}

La figura 7 mostra la copertina del libro pubblicato in portoghese (BR) il 30 settembre 2020. Dalla sua pubblicazione, il libro ha accumulato un totale di 715 visualizzazioni. 
Figura 7 - Mostra la copertina del libro pubblicato in portoghese (BR) il 30 settembre 2020.

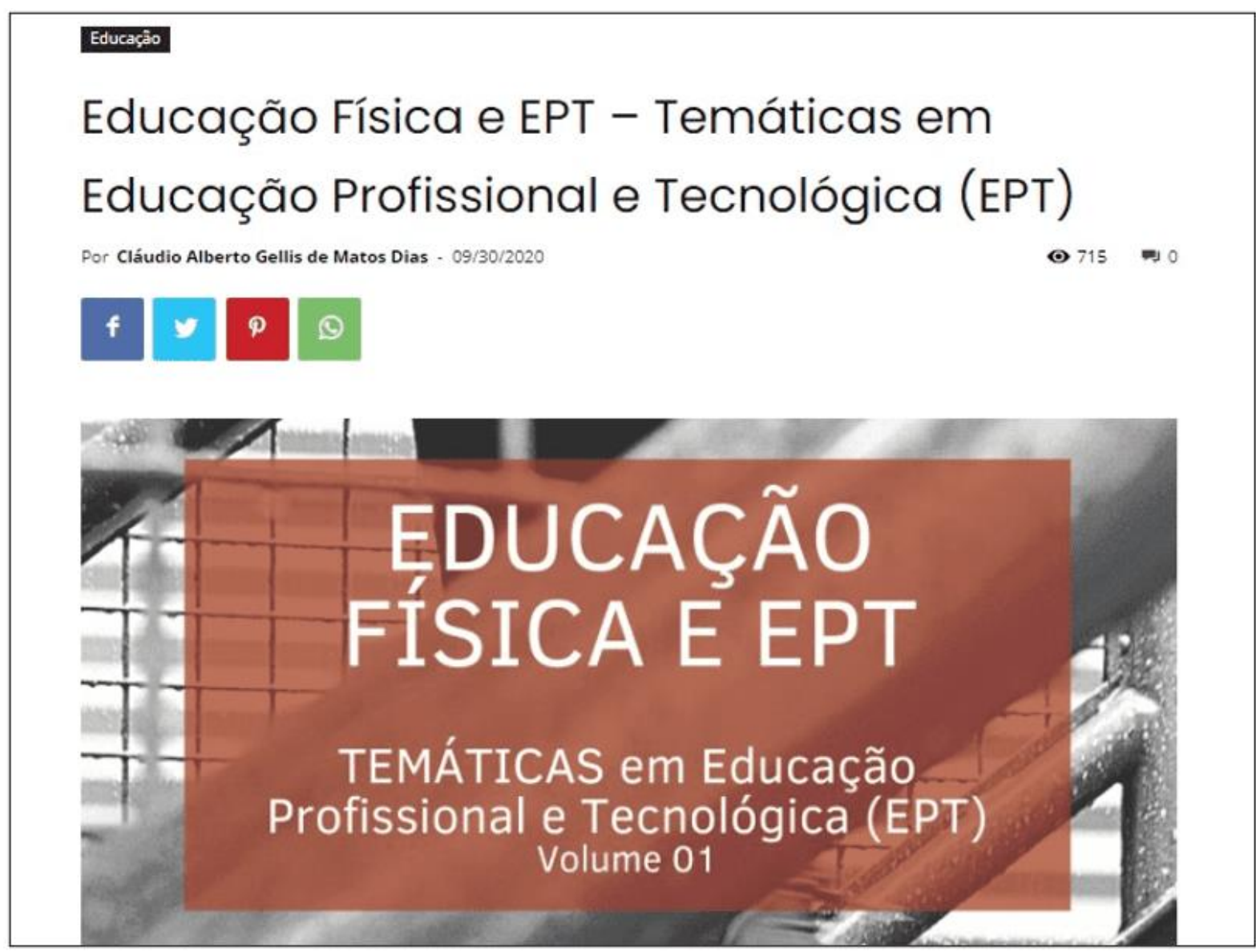

Fonte: Adattato da Viana e Dias (2020).

Il libro pubblicato in inglese (versione del libro in portoghese) il 10 dicembre 2020 accumula da allora 248 visualizzazioni (Figura 8). 
Figura 8 - Mostra la copertina del libro pubblicato in inglese il 10 dicembre 2020.

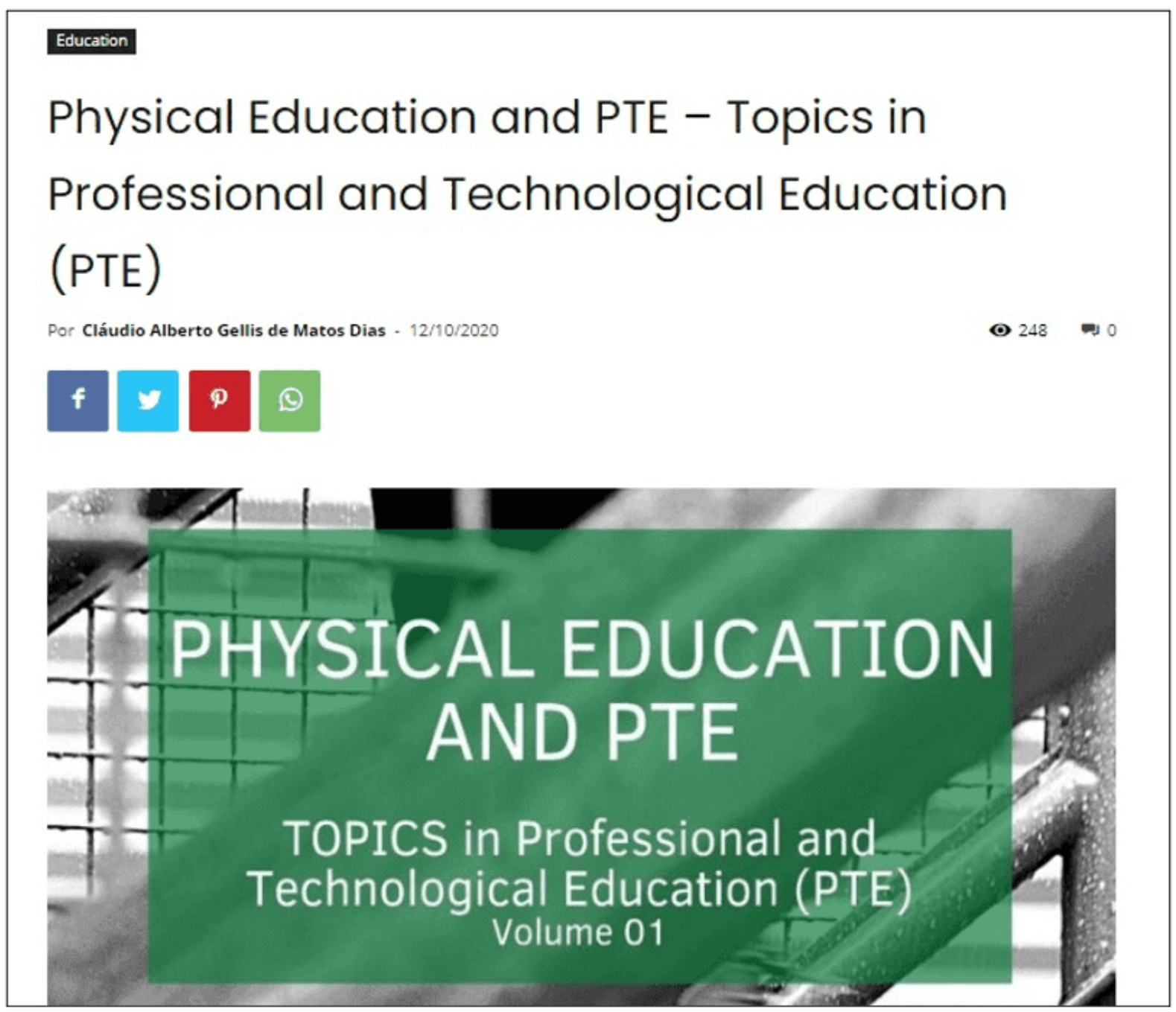

Fonte: Adattato da Viana e Dias (2020b).

\section{ARTICOLI PUBBLICATI SU RIVISTE INDICIZZATE}

L'articolo pubblicato sulla rivista Research, Society and Development (Qualis 20132016 B2 e Qualis reference A3) il 2 luglio 2020 aveva, secondo le informazioni degli editori, 91 visualizzazioni (Figura 9). 
Figura 9 - Mostra la copertina dell'articolo pubblicato sulla rivista Research, Society and Development il 2 luglio 2020.

\begin{tabular}{|} 
Research, Society and Development, v. 9, n. 8, e239985704, 2020 \\
(CC BY 4.0) | ISSN 2525-3409 | DOI: http://dx.doi.org/10.33448/rsd-v9i8.5704 \\
Quadras de esportes em condições de uso adequado no Brasil: \\
influência no ensino-aprendizagem nas aulas de Educação Física (2015) \\
Sports courts in conditions of proper use in Brazil: influence on teaching-learning in \\
Physical Education classes (2015) \\
Canchas deportivas en condiciones de uso adecuado en Brasil: \\
influencia en la enseñanza-aprendizaje en las clases de Educación Física (2015) \\
Recebido: 16/06/2020 | Revisado: 18/06/2020 | Aceito: 22/06/2020 | Publicado: 02/07/2020 \\
Valderi Nascimento Viana \\
ORCD: https:/orcid.org/0000-0002-0885-5834 \\
Instituto Federal do Amapá, Brasil \\
E-mail: wallderinascimento@gmail.com
\end{tabular}

La figura 10 mostra la copertina del manuale sull'applicazione EFE pubblicato sulla Revista Multidisciplinar Núcleo do Conhecimento (Qualis reference B3) il 14 aprile 2021. La pubblicazione in portoghese aveva 244 visualizzazioni. 
Figura 10 - Mostra la copertina del manuale sull'applicazione EFE pubblicato sulla Revista Multidisciplinar Núcleo do Conhecimento il 14 aprile 2021.

\section{Educaçâo Fisica}

MANUAL DE PRATICAS EM EDUCAÇÃO FÍSICA NA EDUCAÇÃO PROFISSIONAL E TECNOLÓGICA UTILIZANDO O APLICATIVO GRATUITO EFE - EPTFIS

Por Carla Dendasck - RC: 82194 - 14/04/2021

DOI: $10.32749 /$ nucleodoconhecimento.com.br/educacao-fisica/manual-de-praticas

\section{ARTIGO ORIGINAL}

VIANA, Valderi Nascimento [1], FECURY, Amanda Alves [2], OLIVEIRA, Euzébio de [3], DENDASCK, Carla Viana [4], DIAS, Claudio Alberto Gellis de Mattos [5]

Fonte: Adattato da Viana et al. 2021 - 2021.

II manuale è stato pubblicato in altre sei lingue, oltre alla lingua portoghese. La tabella 1 mostra il numero di accessi (e l'URL) al manuale sull'applicazione EFE pubblicato sulla Revista Multidisciplinar Núcleo do Conhecimento fino al 14 giugno 2021, per lingua. In totale, dalla pubblicazione al 14 giugno 2021 ci sono stati 374 visualizzazioni. 
Tabella 1 - Mostra il numero di accessi (e url) al manuale sull'applicazione EFE pubblicato sulla Revista Multidisciplinar Núcleo do Conhecimento fino al 14 giugno 2021, per lingua.

\begin{tabular}{|c|c|c|}
\hline IDIOMA & $\begin{array}{c}\text { NÚMERO } \\
\text { DE } \\
\text { ACESSOS }\end{array}$ & LINK DE ACESSO \\
\hline PORTUGUES & 244 & $\begin{array}{l}\text { https://www.nucleodoconhecimento.com.br/e } \\
\text { ducacao-fisica/manual-de-praticas }\end{array}$ \\
\hline INGLES & 20 & $\begin{array}{l}\text { https://www.nucleodoconhecimento.com.br/e } \\
\text { ducation-physics-en/manual-of-practices }\end{array}$ \\
\hline ESPANHOL & 24 & $\begin{array}{l}\text { https://www.nucleodoconhecimento.com.br/e } \\
\text { ducacion-fisica-es/manual-de-practicas }\end{array}$ \\
\hline FRANCÊS & 23 & $\begin{array}{l}\text { https://www.nucleodoconhecimento.com.br/e } \\
\text { ducation-physics-fr/manuel-de-pratiques }\end{array}$ \\
\hline ITALIANO & 26 & $\begin{array}{l}\text { https://www.nucleodoconhecimento.com.br/e } \\
\text { ducazione-fisica-it/manuale-di-pratiche }\end{array}$ \\
\hline ALEMÃO & 21 & $\begin{array}{l}\text { https://www.nucleodocon hecimento.com.br/b } \\
\text { ildung-physik-de/handbuch-der-praktiken }\end{array}$ \\
\hline RUSSO & 16 & $\begin{array}{l}\text { https://www.nucleodoconhecimento.com.br/o } \\
\text { бразование-физика-ru/пособие-по-практике, }\end{array}$ \\
\hline TOTAL & 374 & \\
\hline
\end{tabular}

Fonte: Adattato da Viana et al. (2021), Viana et al. (2021b), Viana et al. (2021c), Viana et al. (2021d), Viana et al. (2021e), Viana et al. (2021f), Viana et al. (2021g).

\section{DISCUSSIONE}

All'interno dell'ambiente scientifico, tutta la teoria e la metodologia cercano di avvicinare lo studente alle conoscenze prodotte dalla scienza. Nell'ambito del processo attraverso la formazione, il contatto con la scrittura di articoli, la costruzione di siti web e l'applicazione, la costruzione di ricerche, analisi e diffusione dei dati, si sono rivelati di grande importanza. In ogni produzione si sta ricercando una realtà, che stabilisce un legame con le situazioni e i contesti socioculturali. La contestualizzazione 
della conoscenza è uno strumento per un apprendimento significativo. È opportunistica, nuova esperienza o di dimissioni delle conoscenze, è sviluppare la capacità di analizzare, ipotesi, senso critico all'interno di una struttura attuale, poiché avviene l'interazione con fatti e fenomeni della vita quotidiana (Yamaguchi e Furtado, 2019).

Tutti i materiali pubblicati, fisicamente o on-line, fungonoda mezzo di diffusione scientifica. Nel caso dell'applicazione tutti i contenuti trattati si basano su conoscenze scientifiche, con un accesso facile e semplice. L'insegnante acquisisce nuove conoscenze e può essere messo in pratica allinterno della scuola in tempo reale. Secondo Rossi (2019)

Resta da avvicinare la scienza degli articoli e dei congressi alla vita dei brasiliani che tanto hanno bisogno di quasi tutto per quanto riguarda la salute, la qualità della vita, la sostenibilità, la mobilità, gli usi della tecnologia e la cittadinanza.

Così, sia a livello teorico (articoli, libri, sito web) che l'applicazione dell'app servono da stimolo all'esperienza scientifica, è pensare alla scienza articolata con realtà, accesso e linguaggio accessibile a chiunque cerchi conoscenza. La divulgazione va oltre l'assunzione di una conoscenza sistematizzata, serve anche a deliziare, motivare e stimolare l'altro. (Rossi, 2019).

Nell'educazione fisica, la visione di una pratica affinata nella tecnica dalla tecnica o dalle prestazioni sportive è ancora molto forte. Le prestazioni e la tecnica devono essere considerate dal docente, ma non considerate prioritarie e devono essere all'interno di uno standard di eccellenza senza rispettare l'individualità dello studente. In una prospettiva di formazione globale, le classi di educazione fisica devono lavorare il loro oggetto di studio in un'ampia dimensione, poiché gli studenti sono inseriti all'interno di una società che ha una cultura e che produce significati, oltre a rivelare contraddizioni di comportamenti, atteggiamenti che devono essere affrontati / considerati (Coutinho, 2010; Viana et al., 2020). 
Così, con produzioni scientifiche basate sulle scienze umane e sociali contribuiscono solo all'area dell'educazione fisica, superando solo l'uso delle scienze della salute, c'è la possibilità di porre l'educazione fisica allo stesso livello di rilevanza e importanza di altre componenti del curriculum scolastico (Balbino e Urt, 2018). Durante il processo di ricerca, articoli e libri sono stati tradotti in altre lingue. Questo atteggiamento è importante per ampliare il campo di applicazione della ricerca, e la produzione può essere correlata alla letteratura nazionale e internazionale, mostrando una capacità di comunicazione, accesso, cooperazione e supporto con ciò che viene prodotto dal mondo nell'ambito dell'educazione fisica (Coutinho, 2010).

Per quanto riguarda la produzione scientifica al Nord, Pereira e Silveira (2019) hanno effettuato un'analisi della produzione scientifica di ricercatori della Regione Settentrionale al Meeting Nazionale della Ricerca nell'Educazione Scientifica e hanno riferito che il Nord ha la minima quantità di ricerche presenti all'evento. Lo studio mostra anche che Amapá non ha svolto alcuna presentazione e lavoro nelle edizioni 2009, 2011, 2013, 2015 e 2017. Secondo lo studio, il maggior numero o il più piccolo di produzioni è legato alla località del campus universitario, poiché sono responsabili della maggior parte delle attività scientifiche. A conferma del suddetto studio, Sidone et al. Altre regioni come il Nordest $15 \%$ e il Midwest e il Nord insieme non hanno raggiunto il $10 \%$ del totale nazionale. Oltre alla concentrazione delle università nelle regioni del Sud e del Sud-Est, un altro fattore sottolineato a questa produzione è la maggiore disponibilità di risorse umane.

\section{CONCLUSIONI}

Durante il master, la produzione scientifica in varie forme si è rivelata necessaria durante il processo di formazione. Unire la teoria alla pratica della scrittura, ha facilitato la produzione della tesi che dovrebbe essere presentata alla fine del corso. Attraverso il processo di organizzazione, la pianificazione delle idee ha portato a un nuovo pensiero strutturato secondo gli standard dell'accademia, ma che non si limita a questo spazio e alle sue norme. 
Avere un contributo che va oltre la scrittura scientifica, durante questo percorso di apprendimento è stata ampiamente, cioè la creazione di applicazioni, siti web, libri, articoli, metodologie di pratiche pedagogiche. Tutta questa esperienza avrà un impatto positivo sul futuro come ricercatore e professionista dell'educazione fisica.

In questo contesto, tutta la produzione durante la laurea magistrale contribuisce anche quantitativamente e qualitativamente alla produzione scientifica della regione settentrionale, impre un impatto positivo su di essa e sul programma del master. Oltre all'importanza nel contributo della creazione e diffusione scientifica, le produzioni tendono ad avere un impatto positivo sulla valutazione e sul mantenimento del programma PROFEPT-IFAP, svolto da CAPES.

\section{RIFERIMENTI}

BALBINO, S. I.; URT, S. D. C. Prática Pedagógica em Educação Física para a Educação Integral em Tempo Integral. Pensar a Prática, v. 21, n. 4, p. 775-785, 2018.

CAPES. Ficha de Avaliação - Programas Acadêmicos e Profissionais Área 46: Ensino. Brasília DF, 2020. Disponível em: < https://www.gov.br/capes/pt-br/centraisde-conteudo/FICHA_ENSINO.pdf >. Acesso em: 14 Jun 2021.

COUTINHO, R. X. A influência da Produção Científica nas Práticas de Professores de Educação Física, Ciências e Matemática em Escolas Públicas Municiais de Uruguaiana - RS. 2010. 94p. (Mestrado). Universidade Federal de Santa Maria, Santa Maria RS.

FALASTER, C.; FERREIRA, M. P.; GOUVEA, D. M. R. D. O efeito da publicação científica do orientador na publicação dos seus orientados. Revista de Administração Contemporânea, v. 21, n. 4, p. 458-480, 2017.

GOOGLEANALYTICS. Google Analytics Aplaticativo Oficial EFE - Pagina Inicial. 2021.

Disponível

em: 
https://analytics.google.com/analytics/web/?authuser=1\#/p265457828/reports/default home >. Acesso em: 14 Jun 2021.

MACHADO, M. F. R. C.; URBANETZ, S. T. Mestrado profissional em educação profissional e tecnológica no Instituto Federal do Paraná: desafios e expectativas. Atos de Pesquisa em Educação, v. 14, n. 2, p. 885-903, 2019.

MAIA, R. T. A importância da disciplina de metodologia científica no desenvolvimento de produções acadêmicas de qualidade no nível superior. Revista Urutágua, v. 14, n. 1, 2008.

PEREIRA, T. Z. M.; SILVEIRA, C. A Produção Acadêmica Da Região Norte: Uma Análise Na Ata Do Xi Encontro Nacional De Pesquisa Em Educação Em Ciências. REAMEC - Rede Amazônica de Educação em Ciências e Matemática, v. 7, n. 2, p. 245-260, 2019.

PLAYCONSOLE. Google Play Console Painel - EFE EduFIsEPT. 2021. Disponível em:

https://play.google.com/console/u/0/developers/4760644030222400117/app/497210 7118591571772/app-dashboard?timespan=thirtyDays\&showKpiMenu=null >. Acesso em: 14 Jun 2021.

RMNC. Revista Multidisciplinar Nucleo do Conhecimento. São Paulo SP, 2021. Disponível em: < https://www.nucleodoconhecimento.com.br/ >. Acesso em: 14 Jun 2021.

ROSSI, A. V. Sobre A Valorização Das Contribuições Da Divulgação Científica No Brasil. In: VOIGT, C. L. (Ed.). O ensino de química. Ponta Grossa PR: Atena Editora, v.1, 2019. cap. 11, p.136-148.

RSD. Research, Society and Development. Vargem Grande paulista SP, 2021. Disponível em:<https://rsdjournal.org/index.php/rsd >. Acesso em: 14 Jun 2021. 
SIDONE, O. J. G.; HADDAD, E. A.; MENA-CHALCO, J. P. A ciência nas regiões brasileiras: evolução da produção e das redes de colaboração científica. Transinformação, v. 28, n. 1, p. 15-32, 2016.

URBANETZ, S. T.; CASSIANO, E. L.; BETTONI, V. O Mestrado Profissional Em Educação Profissional E Tecnológica-Profept-E O Significado Dessa Oferta De Formação Em Pós Graduação No Brasil. Movimento-revista de educação, v. 7, n. 14, 2020.

VIANA, V. N.; DIAS, C. A. G. D. M. Educação Física e EPT - Temáticas em Educação Profissional e Tecnológica (EPT). Macapá AP: 2020. 45 p. ISBN 97865-86069-90-7. Disponível em:

https://www.nucleodoconhecimento.com.br/livros/wp-content/uploads/2020/09/livroeducacao-Fisica-e-EPT-educacao-profissional-e-tecnologica-2020.pdf >.

. Physical Education and PTE - Topics in Professional and Technological Education (PTE). Macapá AP: 2020b. 45 p. ISBN 978-65-86069-89-1. Disponível em: < https://www.nucleodoconhecimento.com.br/livros/en/education/physicaleducation-and-pte $>$.

VIANA, V. N. et al. Quadras de esportes em condições de uso adequado no Brasil: influência no ensino-aprendizagem nas aulas de Educação Física (2015). Research, Society and Development, v. 9, n. 8, p. e239985704, 2020. Disponível em: < https://rsdjournal.org/index.php/rsd/article/view/5704/4727 >.

VIANA, V. N. et al. Manuel de pratiques en éducation physique en éducation professionnelle et technologique utilisant l'application gratuite EFE - EPTFIS. Revista Científica Multidisciplinar Núcleo do Conhecimento, v. 5, p. 4, 2021d. Disponível em: < https://www.nucleodoconhecimento.com.br/education-physics-fr/manuel-depratiques $>$.

- Manuale di pratiche in educazione fisica nell'educazione professionale e tecnologica utilizzando l'applicazione gratuita EFE - EPTFIS. Revista Científica 
Multidisciplinar Núcleo do Conhecimento, v. 5, n. 4, p. 65-76, 2021e. Disponível em: < https://www.nucleodoconhecimento.com.br/educazione-fisica-it/manuale-dipratiche $>$.

Handbuch der Praktiken im Sportunterricht im beruflichen und technologischen Unterricht mit der kostenlosen Anwendung EFE - EPTFIS. Revista Científica Multidisciplinar Núcleo do Conhecimento, v. 5, n. 4, p. 6576, 2021f. Disponível em: < https://www.nucleodoconhecimento.com.br/bildungphysik-de/handbuch-der-praktiken $>$.

. Пособие по практике физического воспитания в профессиональном и технологическом образовании с использованием бесплатного приложения EFE EPTFIS. Revista Científica Multidisciplinar Núcleo do Conhecimento, v. 5, n. 4, p. 65-76, 2021g. Disponível em: < https://www.nucleodoconhecimento.com.br/образование-фризика-ru/пособие-попрактике >.

VIANA, V. N. et al. Manual De Praticas Em Educação Física Na Educação Profissional E Tecnológica Utilizando O Aplicativo Gratuito EFE - EPTFIS. Revista Científica Multidisciplinar Núcleo do Conhecimento, v. 5, n. 4, p. 65-76, 2021. Disponível

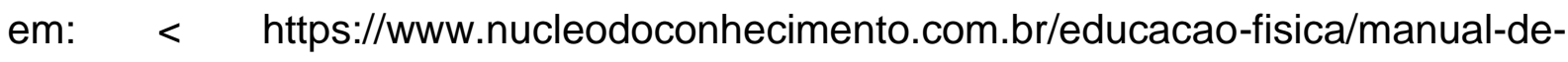
praticas $>$.

. Manual Of Practices In Physical Education In Professional And Technological Education Using The Free Application EFE - EPTFIS. Revista Científica Multidisciplinar Núcleo do Conhecimento, v. 5, n. 4, p. 65-76, 2021b. Disponível em: < https://www.nucleodoconhecimento.com.br/education-physics-en/manual-ofpractices $>$.

- Manual De Prácticas En Educación Física En Educación Profesional Y Tecnológica Utilizando La Aplicación Gratuita EFE - EPTFIS. Revista Científica Multidisciplinar Núcleo do Conhecimento, v. 5, n. 4, p. 65-76, 2021c. Disponível 
em: < https://www.nucleodoconhecimento.com.br/educacion-fisica-es/manual-depracticas $>$.

YAMAGUCHI, K. K. D. L.; FURTADO, M. A. S. A Escrita Científica Como Aprendizagem Contextualizada: Uma Abordagem Metodológica A Partir De Um Experimento De Mistura De Cores In: VOIGT, C. L. (Ed.). O ensino de química. Ponta Grossa PR: Atena Editora, v.1, 2019. cap. 12, p.149-158.

Inviato: Giugno 2021.

Approvato: Giugno 2021. 